\title{
LA INVESTIGACIÓN EN GESTIÓN DE EMPRESAS: FUNDAMENTAL Y REALIDAD
}

AUTORES: Merchán Ponce Narda ${ }^{1}$

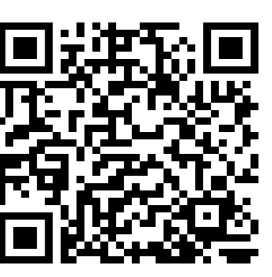

DIRECCIÓN PARA CORRESPONDENCIA: (1 km vía Noboa Campus los ángeles)

Fecha de recepción: 13/01/2019

Fecha de aceptación: 21/04/2019

\section{RESUMEN}

Cuál es el propósito final de la investigación y gestión de empresas? ¿Tiene esta investigación una base racional y rigurosa? ¿Con investigación de calidad, relevante y arraigada en la realidad de los procesos observados? Este artículo tiene el desarrollo de alcanzar y analizar objetivos en cuestiones interdependientes acerca de la investigación en gestión de empresas, aplicando la metodología y sistematizar el desarrollo de la gestión empresarial en el logro de mejores resultados. Recordemos que la empresa es una organización productiva que pretende conseguir la asignación de los recursos de la empresa lo más eficientemente posible tales como el debate a su razón de ser y sus mitos racionales, su verdadera utilidad y contribución social, al valor de los resultados y su vinculación con la realidad de las empresas en los análisis a los elementos económicos y sociales.

PALABRAS CLAVE: Contribución social, organización, objetivo, procesos, relevancia

\section{STUDENT PERMANENCE IN HIGHER EDUCATION: THE VISION OF THE TEACHER}

\begin{abstract}
What is the ultimate purpose of research and business management? Does this research have a rational and rigorous basis? With quality research, relevant and rooted in the reality of the processes observed? This article has the development of reaching and analyzing objectives in interdependent questions about research in business management, applying the methodology and systematizing the development of business management in the achievement of better results. Recall that the company is a productive organization that aims to achieve the allocation of resources of the company as efficiently as possible such as the debate to its rationale and its rational myths, its true utility and social contribution, the value of results and its link with the reality of the companies in the analysis of the economic and social elements.
\end{abstract}

\footnotetext{
${ }^{1}$ Merchán Ponce Narda, Docente Universidad Estatal del Sur de Manabí, Ecuador Jipijapa-Manabí
} 
Merchán Ponce Narda.

KEYWORDS: social contribution, organization, objective, processes, relevance

\section{INTRODUCCIÓN}

La actividad investigadora es, cada vez en más países del mundo, una de las principales componentes de la profesión del docente e n e 1 ámbito de la dirección y gestión de empresas para que resulte paradójico la legitimidad y el reconocimiento de esta actividad en lo absoluto de una cuestión obvia. De hecho, fuera del ámbito académico es una actividad desconocida, en el mejor de los casos ya que en el peor, suele despertar

recelos entre algunos docentes, tales como los directivos ${ }^{1}$, los profesionales de la consultoría y otros grupos de referencia a los académicos. Debido a ello son diversas las preguntas relevantes que podían plantearse al respecto:

¿Cuál es el propósito final de la investigación en gestión de empresas?

¿Es la investigación una base racional y rigurosa? ¿Es investigación de calidad, relevante y arraigada en la realidad de los métodos observados? ¿Es la investigación guiada por las necesidades sociales o por otros intereses? Todas estas alusiones de cuestiones son relativamente comunes y cotidianas en charlas de pasillo, aulas y otros intercambios informales entre colegas, pero parece que tienden a ocupar un lugar marginal o incluso son silenciadas en lo académico y convencionales, tales como demuestran muchas revistas académicas. Sin embargo, como apunta Gill (2015), un debate riguroso que supere la tendencia tanto a la autoindulgencia, al lamento que resulta pertinente de cara a avanzar en una perspectiva de análisis o demanda (política) para el cambio.

La gestión de empresas establecerá las interrelaciones entre los principios de la organización en concordancia con el número de los procesos funcionales. Se deben tener en cuenta factores como cuál es el objetivo principal de la empresa, determinar las actividades que se realizarán, asignar el personal idóneo, dividir e integrar las actividades entre los empleados de la empresa y fomentar el grupo social productivos a los bienes y servicios mediante la administración y la gestión de todos sus elementos.

En este artículo se ha optado por utilizar el masculino genérico por motivos del lenguaje y fluidez al mismo.

\section{OBJETIVOS DE LA ORGANIZACIÓN EMPRESARIAL}

Al encontrar investigadores capaces de llevar a cabo investigaciones cualitativas o de evaluar de forma consistente y fiable trabajos basados en enfoques cualitativos, y los procesos sean mucho más rápido, pero que no se arrepentía de haber hecho una investigación cualitativa empresarial más detallada, debido al "conocimiento real" que le había aportado a la empresa. Parece ser que los estudios cuantitativos pese a que no suelen proporcionar un contacto directo con el terreno o el campo del desarrollo empresarial, tienen una apariencia de mayor rigor y son hegemónicos en las publicaciones de revistas de prestigio, ofreciendo, por consiguiente, mejores oportunidades de publicación empresariales y fomentar su desarrollo. Un colega nos señaló en un 
congreso académico que su "trabajo de campo", había consistido esencialmente en un "apartado de correos" donde recibía las respuestas a los cuestionarios empresariales una vez tabulados y los sometía a tratamientos estadísticos sofisticados (y cabría apuntar que, con el desarrollo de los cuestionarios administrados en línea, ni siquiera esa fase más tangible de los "trabajos de campo" parece tener mucho futuro). Con las evidencias consignadas que nos llevan a plantear la existencia de un mito a la gestión empresarial al futuro.

\section{LA GESTIÓN Y EL ANÁLISIS DE DATOS}

El análisis de la información que se obtuvo esta por lo general condicionada al cumplimiento de modelos o teorías establecidas en lugar de explicar claramente las realidades a menudo complejas y contradictorias de las que se recogieron en el trabajo de campo, cuando este existe. Estas realidades no coinciden muchas veces con las expectativas y las orientaciones de parte importante de las publicaciones académicas que tienden a favorecer la adaptación de los hechos a la teoría y no al revés. Por ejemplo, los datos empíricos cuantitativos resultan esenciales en la investigación, en ocasiones pueden ser susceptibles de recibir ciertos "retoques estadísticos" a lo largo del proceso en el cual varios modelos o enfoques alternativos son testeados en un proceso de prueba y error hasta que, finalmente, agotadas muchas veces la mayoría de las relaciones que parecían de salida más lógicas y obvias, ciertas relaciones parecen "funcionar".

Una vez constatado que el modelo funciona, la labor del investigador en gestión es seguir esta corriente dominante o mainstream, se puede centrar en redactar de la forma más sofisticada posible la discusión de las relaciones "detectadas". Y las decisiones de gestión rara vez hay errores estadísticos y conceptuales que precedieron a la tabulación de los resultados aparentemente coherentes y racionales. Obviamente, no se puede afirmar que se trate de una práctica generalizada, ni se debe ignorar, desde nuestra perspectiva, la calidad y la fiabilidad de muchas investigaciones cuantitativas publicadas en revistas académicas, pero tampoco se debería pasar por alto una crítica que, pese a no tener mucha presencia en nuestras revistas, es, una experiencia, compartida por una parte muy considerable de los académicos empresariales en este ámbito. Los métodos cualitativos o etnográficos pueden también dar lugar a una interpretación de los datos que tiende a confirmar los modelos teóricos de los que se parte como premisa. Sin embargo, por su carácter más inductivo y por la riqueza de la información recogida, estos métodos, centrados en el análisis de los resultados obtenidos por el investigador empresarial en contacto directo con el objeto de estudio y directamente enraizados en el campo de la investigación, pueden facilitar el desarrollo de nuevas teorías y nuevas conceptualizaciones fruto del proceso de análisis a la realidad que se observa, si las teorías previas no se contrastan y resultan adecuadas vienen los resultados obtenidos. 
Merchán Ponce Narda.

\section{LA RELACIÓN DE LAS GESTIONES ACADÉMICAS EMPRESARIAL}

El proceso de relación es el propósito principal del desarrollo a la investigación en gestión. Por un lado, y la preocupación por la legitimidad científica de nuestra comunidad investigadora empresarial han posibilitado la tendencia a la presentación de artículos investigativos cada vez más "técnicos y sofisticados".

Así, cada vez se hace un mayor hincapié en los aspectos metodológicos y en especial en la introducción de modelos cuantitativos sofisticados. Estos elementos dan una mayor apariencia de racionalidad y rigor de los trabajos realizados, sin tener que mejorar ni la pertinencia, ni la relevancia, ni el rigor y la exactitud de las interpretaciones realizadas. En última instancia, la profusión de fórmulas técnicas y complejas que se aplican a conjuntos de datos incompletos, poco fiables o mal tratados, tiende a transmitir una suerte de imagen de "ciencia ficción", con sus propios "efectos especiales", de forma que se burlan del lector cuando tratan de trasmitirle la idea de que se trata de conocimiento científico libre de toda subjetividad, donde todas las variables parecen estar controladas, tratando de imitar a las ciencias duras. Este torpe mimetismo bebe del anhelo de las ciencias económicas - la hermana mayor y reputada de las ciencias de dirección y gestión - de simular o aparentar la seriedad de disciplinas científicas duras, huyendo de su "pobre" realidad de ciencia social, cuestión ésta que ha sido apuntada por diversos autores del área de las ciencias económicas para dicho ámbito (ver, por ejemplo, Mishan, 1969; Krugman, 1999).

Por otro lado, el énfasis de la mayoría de las revistas académicas gestión en las contribuciones teóricas, más que en una preocupación por la verdad conduce a una escritura aséptica, que cumple con los requerimientos del paradigma y la literatura dominante. Este contexto no promueve la gestión de artículos críticos, que están fuera de la ortodoxia o corriente principal, pero que pueden tratar de aprehender la realidad de la investigación de campo. Más bien, se anima a una especie de "fuga en la abstracción" en la que los mismos modelos y las mismas teorías que se supone representan la realidad parecen infinitamente recicladas y se retroalimentan sin fin.

Así es como un "ámbito de investigación" de las ciencias de la administración se crea y recrea con su propia lógica interna desconectándose cada vez más de la realidad. Y se trata de una lógica prácticamente global, puesto que son cada vez más los países del mundo Europa y diversos países africanos que se han unido a este paradigma que prioriza la importancia de las publicaciones en el ámbito de la gestión empresarial, habiéndose producido un desarrollo en el ámbito de investigación en gestión, con una enorme competencia global por publicar en las mejores revistas pero que, paradójicamente, no ha incidido en el surgimiento reseñable de teorías innovadoras y de impacto (Alvesson y Sandberg, 2013).

En este punto se puede reflexionar brevemente, acerca de los efectos a esta lógica. Así como en la teoría económica una teoría de ideas que se basa en premisas erróneas puede tener su efecto al ser aplicada a la realidad, el supuesto debate de ideas que se genera en el área de las ciencias de la gestión con toda su sofisticación

132 UNESUM-Ciencias. Publicación cuatrimestral. Vol. 3, Año 2019, No. 1 (abril) 
y apariencia de relevancia y rigor, en gran medida más allá de la pérdida de recursos que se plantea para la sociedad.

Un espectáculo que en muchos casos es virtual e intangible: parte de datos que los investigadores, cada vez con mayor frecuencia, no obtienen visitando empresas o plantas de producción para encuestas o entrevistas, sino de un proceso de "trabajo de campo" en línea, en el mejor de los casos, o de bases de datos que adquieren pagando grandes sumas (procedentes en la mayor parte de los casos de recursos públicos), datos que elaboran con complejos programas de análisis estadístico informático, que, una vez analizados en artículos que son redactados en procesadores de textos. Pero al parecer en el ámbito "real" en la gestión de empresas a supuestos grupos de interés prioritarios (directivos, empleados y decisores públicos, entre otros), rara vez consultan dichas publicaciones y cuando lo realizan no parecen otorgarles demasiada consideración y utilidad (Mintzberg, 2004).

Al tratar de establecer una vinculación directa con el ámbito empresarial, mientras que el proceso de selección implementado tiende a eliminar con celeridad este tipo de trabajos y a reproducir publicaciones cada vez más sofisticadas, opacas y alejadas de la realidad del día a día de las empresas y de otras organizaciones.

\section{MARCO METODOLÓGICO}

Metodología analítica: Se utilizó este método de investigación el cual nos permitió estudiar la realidad de las empresas a la ejecución de las encuestas a los empleados de las organizaciones.

Metodología descriptiva: Este método de investigación se utiliza para detallar cada proceso describiendo todos los riesgos y las actividades que los empleados de la en las distintas organizaciones.

Método bibliográfico: Este método permite sintetizar los diferentes aspectos conceptuales teóricos, relacionadas con las variables de la investigación.

Método estadístico: Fue utilizado para que nuestra investigación obtenga enfoques

Estadísticos a través de resultados los cuales se manifiestan en gráficos, en el proceso de recolecta y se pone la información en porcentajes cuyos niveles serán de mínimo a Máximo.

\section{DISCUSIÓN Y CONCLUSIONES}

En la mayor parte de los casos, se lleve a cabo de buena fe y en condiciones difíciles, por parte de investigadores respetables, que, probablemente, obtendrán una utilidad y unos beneficios reseñables para otros aspectos de su actividad profesional como, por ejemplo, la actividad docente. 
Merchán Ponce Narda.

En la perspectiva a la visión crítica monolítica, que es exteriorizada por algunos críticos con la investigación en nuestro ámbito, no se debe juzgar la actividad investigadora en el ámbito empresarial de forma homogénea, puesto que la calidad y la pertinencia de las publicaciones no lo es. Sin embargo, se entiende que el debate a la relevancia y los efectos perversos de los mitos racionales de la investigación en gestión que se ha tratado en el resumen de este artículo puede contribuir a reforzar la calidad y la fundamentación en la realidad de la investigación científica en el área de la gestión de empresas. L o qu e resulta difícil, dadas las limitaciones de extensión del trabajo, entrar a discutir a profundidad cómo se podría limitar la influencia negativa de los mitos analizados que en la actualidad se encuentran ampliamente institucionalizados en un conjunto de prácticas, normas, y organizaciones académicas de creencias que pueden ser útil para compartir algunas consideraciones optimistas finales en el tema.

El interés relacionado con la investigación en gestión de empresas, pueden resultar útiles a la sociedad que siguen poco o nada transitadas. Lejos de las multitudes, los ámbitos temáticos están saturados por la acumulación de numerosos trabajos, con una mayor libertad de acción para los investigadores que luego son menos dependientes de los dictados de los paradigmas dominantes y de sus más ortodoxos defensores.

Exponiendo ciertos enfoques metodológicos como la teoría anclada (Strauss y Corbin, 1990) o la investigación de acción (Greenwood and Levin, 2006), pueden ayudar a prevenir o limitar los excesos y fortalecer los vínculos entre la teoría y la investigación de campo. Además, parece que las revistas que publican artículos con contenidos relativos a la dirección y gestión de empresas al no ser incluidas en dichas categorías proliferando en los últimos años a darse un aumento en las posibilidades de publicación de artículos que se salen del patrón único modelado por los mitos racionales dominantes.

Por 10 tanto, se puede optar por otras alternativas que quizá puedan ser menos prestigiosas, pero más abiertas, y en ocasiones pueden tener una difusión y un impacto equivalente o superior a aquellas. Este es el caso, por ejemplo, de numerosas revistas más o menos especializadas en el campo del desarrollo sostenible que se crearon en los últimos años.

Otra forma de limitar la influencia de los mitos racionales relacionados con la investigación en gestión consiste en fortalecer los vínculos con las organizaciones y empresas, con los directivos, con los empleados y sus representantes que trabajan en el ámbito de la política de promoción empresarial e industrial, entre otros muchos grupos de interés se pueden presentar los resultados de las investigaciones a los directivos de empresas por ejemplo en el marco de la docencia de los académicos en los programas de formación de postgrado y la formación continua, como es de bien en la esencia de nuestro tejido empresarial, aunque muchas veces no se les dedique la atención que requieren son dos experiencias

134 UNESUM-Ciencias. Publicación cuatrimestral. Vol. 3, Año 2019, No. 1 (abril) 
que se encuentran entre las más relevantes y gratificantes de un académico del ámbito de la gestión de empresas. Estas experiencias que a menudo nos han permitido contrastar los resultados de algunos de nuestros trabajos, recogiendo un feedback que ha resultado ser mucho más riguroso, enriquecedor y, sorprendentemente, mucho más crítico que el que predomina en la literatura académica de gestión, sometimiento a los estándares académicos empresariales para el futuro dominante del ámbito estadístico empresarial.

\section{VALORACIÓN ACERCA DEL ARTÍCULO}

El artículo está dedicado al análisis y a la discusión reflexiva, (al doble sentido del adjetivo) de estas como en otras cuestiones interdependientes, en el análisis de los procesos de la investigación en gestión de empresas, el debate sobre su razón de ser y sobre su verdadera utilidad a la contribución social, al valor en los resultados a su vinculación o no con la realidad de las organizaciones y empresas, como objeto de análisis que se sustenta en la trayectoria académica y personal del autor del artículo y en un amplio análisis de la metodología, basada en métodos de indagación cualitativa y etnográfica, en la realización de entrevistas personales en profundidad a docentes, investigadores, directivos y consultores de empresa.

Es un artículo de corte teórico. Sin embargo, de mantenerse debe cumplir las normas de redacción establecidas para poder declararlo publicable,

\section{REFERENCIAS BIBLIOGRÁFICAS}

Adler, N.J. y Harzing, A.W. (2009). When knowledge wins: Transcending the sense and nonsense of academic rankings. Academy of Management Learning \& Education, 8(1),72-95.

Alvesson, M. y Sandberg, J. (2013). Has management studies lost its way? Ideas for more imaginative and innovative research. Journal of Management Studies, 50(1), 128-152.

ALBURQUERQUE F 2012. La importancia del enfoque del desarrollo económico local. [consulta

14 enero 2017]. Disponible en: http://hdrnet.org/202/1/ALBUQ_La\%20importancia\%20del.pdf

CRUZ CHIMAL J. 2013. Proceso administrativo: planeación, organización, dirección y control. [Consulta 14 enero 2017]. Disponible en: http://www.gestiopolis.com/proceso-administrativo-planeaciónorganización-dirección-y-control/

Evolución histórica de la administración. 2011 [consulta 14 enero 2017]. Disponible e n :

http://administare.blogspot.com/2011/11/evolucion-historica-de-la.html

FUENTES HENRÍQUEZ F 2005. La ciencia de la administración de empresas: Un análisis de sus componentes y de la Contribución de la revista economía y Administración. Revista Economía y Administración No 64.

Disponible

en:

http://www2.udec.cl/ rea/REVISTA\%20PDF/Rev64/art2rea64.pdf 
Merchán Ponce Narda.

Boiral, O. (2003). ISO 9000: Outside the iron cage. Organization Science, 14(6), 720737.

Boiral, O. y Heras-Saizarbitoria, I. (2016). Raison d'être et mythes rationnels de la recherche en gestion. Revue internationale PME, 29(1), pág. 7-15.

Debord, G. (2002). The Society of the Spectacle. Canberra, Hobgoblin Press. Frankfurt, H. (2005).

On Bullshit. Princeton, NJ, Princeton University Press.

Gill, R. M. (2015). Rompiendo el silencio: las heridas ocultas de la universidad neoliberal. Arxius, núm. 32, pág. 45-58.

Glynn, M.A. y Dacin, M.T. (2000). Pluralism and the problem of variety. Academy of Management Review, 25(4), 726-734.

Greenwood, D. J., \& Levin, M. (2006). Introduction to action research: Social research for social change. Thousand Oaks, California: SAGE publications.

Gusdorf, G. (1974). Introduction aux sciences humaines: essai critique sur leurs origines et leur développement. Paris: Les Éditions Ophrys.

Gusdorf, G. (1966). Les sciences humaines et la pensée occidentale: Les origines des sciences humaines (Vol. 2). Paris: Payot.

Hambrick, D.C. (2007). The field of management's devotion to theory: Too much of a good thing? Academy of Management Journal, 50(6), 1346-1352.

Heugens, P. y Mol, M.J. (2005). So, you call that research? Mending methodological biases in strategy and organization departments of top business schools. Strategic Organization, 3(1), 117-128.

Ioannidis, J . P . (2005). Why $\mathrm{mos} t$ published $\mathrm{r}$ e $\mathrm{se}$ a $\mathrm{rch}$ findings are false. PLoS medicine, 2(8), e124, disponible en :

http://journals.plos.org/plosmedicine/article?id=10.1371/journal.pmed.0020124

Kieser, A. y Leiner, L. (2009). Why the rigour-relevance gap in management research is unbridgeable. Journal of Management Studies, 46(3), 516-533.

Krugman, P. (1998). The Accidental Theorist, and other Dispatches from the Dismal Science. New York, NY, Norton.

Miller, A. N., Taylor, S. G. y Bedeian, A. G. (2011). Publish or perish: academic life as management faculty live it. Career Development International, 16(5), 422-445.

Markides, C. (2007): "In search of ambidextrous professors", Academy of Management Journal, 50(4), 762-768.

Mintzberg, H. (2004). Managers, not MBAs: A Hard Look at the Soft Practice of Managing and Management Development. San Francisco: Berrett-Koehler Publishers.

Mishan, E.J. (1969). 21 Popular Economic Fallacies. New York. NY: Praeger.

Morrison, E.W. y Milliken, F.J. (2000). Organizational silence: A barrier to change and development in a pluralistic world. Academy of Management Review, 25(4), 706-725.

136 UNESUM-Ciencias. Publicación cuatrimestral. Vol. 3, Año 2019, No. 1 (abril) 
Kieser, A. y Leiner, L. (2009). Why the rigour-relevance gap in management research is unbridgeable. Journal of Management Studies, 46(3), 516-533.

Pfeffer, J. (2007). A modest proposal: How we might change the process and product of managerial research. Academy of Management Journal, 50(6), 1334-1345.

Power, M. (1997). The audit society: Rituals of verification. OUP Oxford.

Power, M. (2000) "The Audit Society - Second Thoughts", International Journal of Auditing, 4: 111-119.

Rynes, S.L., Bartunek, J.M. y Daft, R.L. (2001). Across the great divide: Knowledge creation and transfer between practitioners and academics. Academy of Management Journal, 44(2), 340-355.

Strauss, A. y Corbin, J.M. (2015). Basics of Qualitative Research: Grounded Theory de Educación. Procedures and Techniques. Londres: Sage Publications, Inc. 
Merchán Ponce Narda.

138 UNESUM-Ciencias. Publicación cuatrimestral. Vol. 3, Año 2019, No. 1 (abril) 